\title{
Biocompatible organic dots with aggregation-induced emission for in vitro and in vivo fluorescence imaging
}

\author{
LI Kai ${ }^{1}$, DING Dan ${ }^{2}$, ZHAO QiuLi ${ }^{3}$, SUN JingZhi ${ }^{3}$, TANG Ben Zhong ${ }^{4,5}$ \& LIU Bin ${ }^{1,2^{*}}$ \\ ${ }^{1}$ Institute of Materials Research and Engineering, 3 Research Link, Singapore 117602 \\ ${ }^{2}$ Department of Chemical and Biomolecular Engineering, National University of Singapore, 4 Engineering Drive 4, \\ Singapore 117576, Singapore \\ ${ }^{3}$ MOE Key Laboratory of Macromolecule Synthesis \& Functionalization, Institute of Biomedical Macromolecules, \\ Department of Polymer Science \& Engineering, Zhejiang University, Hangzhou 310027, China \\ ${ }^{4}$ Department of Chemistry, The Hong Kong University of Science \& Technology, Clear Water Bay, \\ Kowloon, Hong Kong, China \\ ${ }^{5}$ SCUT-HKUST Joint Research Laboratory; State Key Laboratory of Luminescent Materials and Devices; South China University of \\ Technology, Guangzhou 510640, China
}

Received May 17, 2013; accepted June 17, 2013; published online July 27, 2013

\begin{abstract}
Fluorescent probes play a key role in modern biomedical research. As compared to inorganic quantum dots (QDs) composed with heavy metal elements, organic dye-based fluorescent nanoparticles have higher biocompatibility and are richer in variety. However, traditional organic fluorophores tend to quench fluorescence upon aggregation, which is known as aggregationcaused quenching (ACQ) effect that hinders the fabrication of highly emissive fluorescent nanoparticles. In this work, we demonstrate the synthesis of organic fluorescent dots with aggregation-induced emission (AIE) in far-red/near-infrared (FA/NIR) region. A conventional ACQ-characteristic fluorescent dye, 3,4:9,10-tetracarboxylic perylene bisimide (PBI), is converted into an AIE fluorogen through attaching two tetraphenylethylene (TPE) moieties. The fluorescent dots with surface folic acid groups are fabricated from PBI derivative (DTPEPBI), showing specific targeting effect to folate receptor-overexpressed cancer cells. In vivo studies also suggest that the folic acid-functionalized AIE dots preferentially accumulate in the tumor site through enhanced permeability and retention (EPR) effect and folate receptor-mediated active targeting effect. The low cytotoxicity, good FR/NIR contrast and excellent targeting ability in in vitro/in vivo imaging indicate that the AIE dots have great potentials in advanced bioimaging applications.
\end{abstract}

aggregation-induced emission, AIE dots, targeted imaging, fluorescence imaging

\section{Introduction}

In modern bioimaging applications, fluorescent techniques have been proven to be powerful tools in both fundamental research and practical applications [1]. Fluorescent probes play the essential role in bioimaging tasks to ensure desired fluorescence output with high sensitivity, motivating researchers to make great efforts to explore new generations

*Corresponding author (email: cheliub@nus.edu.sg) of probes with high brightness, good photostability and feasible synthetic methods. Among various fluorescent probes, inorganic quantum dots (QDs) have shown great advantages in terms of better photostability and large Stokes shifts as compared to small organic dye molecules [2]. However, the intrinsic components of hazardous heavy metal elements may cause potential toxic issue [3], which needs to be fully understood before using such QD probes for in vivo imaging studies. Moreover, the fluorescence of QDs tends to be diminished in acidic environment [4], compromising the reliability during data analysis. As compared to inorganic 
QDs, various organic dye-loaded fluorescent nanoparticles have shown superior cytocompatibility and fluorescence stability to the QDs. However, conventional organic dyes always suffer weakened or annihilated fluorescence upon aggregation, which is known as aggregation-caused quenching (ACQ) [5]. Hence, the booming development in biomedical applications requires novel class of fluorescent probes with the hope to overcome the shortcomings of the inorganic QD probes and organic dye-loaded fluorescent nanoparticles.

Recently, we have reported several fluorogens that exhibited unique aggregation-induced emission (AIE) characteristics, which are directly opposite to ACQ and will greatly benefit the formulation of highly fluorescent nanoparticles [6, 7]. The AIE fluorogens are non-emissive when they are well-dissolved in solution but become highly emissive upon aggregation due to the restriction of intramolecular rotations (RIR) that activates radiative decay channels [8]. We found that simply attaching an iconic AIE unit, tetraphenylethene (TPE), to various conventional ACQ chromophores will facilitate the conversion to AIE ones, which offers a feasible and efficient approach to synthesize AIE fluorogens [9]. Additionally, using biocompatible distearoyl-sn-glycero-3-phosphoethanolamine-poly(ethylene glycol) (DSPE-PEG) derivatives as the encapsulation matrix, we have demonstrated that the AIE dots greatly outperform inorganic QD probes in in vitro and in vivo long-term cell tracing applications [10]. These results strongly suggest that the AIE fluorogens are promising candidates in the development of next generation of organic fluorescent dots.

In this contribution, we report the fabrication of AIE dots with far-red/near-infrared (FR/NIR) emission and demonstrate their performance in targeted imaging ability using a folate receptor-overexpressed tumor-baring mouse model. Through attaching TPE to a perylene-3,4:9,10-tetracarboxylic bisimide (PBI) derivative with typical ACQ effect, the obtained fluorogen shows pronounced AIE feature with efficient FR/NIR emission upon aggregation. Using the mixture of DSPE-PEG and DSPE-PEG-Folate as the encapsulation matrix, the obtained folate-functionalized AIE dots show higher targeting ability to folate receptor-overexpressed cancer cells and tumors as compared to AIE dots using DSPE-PEG alone during dot fabrication. This study opens a new window to synthesize fluorescent dots with FR/NIR emission and good biocompatibility to benefit the development of advanced imaging probes.

\section{Experimental}

\subsection{Materials}

[ $N, N^{\prime}$-dicyclohexyl-1,7-bis(4'-(1',2',2'-triphenyl)vinyl)phenylperylene-3,4:9,10-tetracarboxylic bisimide] (DTPEPBI) was synthesized according to literature [11]. 1,2-Distearoyl$s n$-glycero-3-phosphoethanolamine- $N$-[methoxy(polyethylene glycol)-2000] (DSPE-PEG 2000 ) was a gift from Lipoid
GmbH (Ludwigshafen, Germany). DSPE-PEG ${ }_{5000}$-Folate was a commercial product of Avanti Polar Lipids, Inc. Tetrahydrofuran (THF), 4',6-diamidino-2-phenylindole (DAPI), 3-(4,5-dimethylthiazol-2-yl)-2,5-diphenyl tetrazolium bromide (MTT), penicillin-streptomycin solution, and trypsinEDTA solution were purchased from Sigma-Aldrich. Fetal bovine serum (FBS) was purchased from Gibco (Lige Technologies, Ag, Switzerland). Milli-Q water was supplied by Milli-Q Plus System (Millipore Corporation, Breford, USA). MCF-7 cells and $\mathrm{H}_{22}$ cells were provided by American Type Culture Collection.

\subsection{Synthesis of DTPEPBI based fluorescent dots}

A THF solution $(0.5 \mathrm{~mL})$ containing $1 \mathrm{mg}$ of DTPEPBI and $2 \mathrm{mg}$ of mixture of DSPE-PEG ${ }_{2000}$ and DSPE-PEG ${ }_{5000^{-}}$ Folate (molar percentage ratio of DSPE-PEG ${ }_{5000}$-Folate was $0 \%$ and $50 \%$, respectively) was poured into $10 \mathrm{~mL}$ of $90 \%$ $(v / v)$ water/THF solution. This was followed by sonication for 60 seconds at $12 \mathrm{~W}$ output using a microtip probe sonicator (XL2000, Misonix Incorporated, NY). The emulsion was then stirred at room temperature overnight to evaporate THF. AIE dots and Folate-AIE dots are assigned to DTPEPBI based fluorescent dots prepared without and with DSPE-PEG $_{5000}$-Folate, respectively. The obtained solution was filtered using a $0.22 \mu \mathrm{m}$ syringe-driven filter to collect the products.

\subsection{Characterization methods}

The UV-Vis spectra of dot suspensions in water were recorded on a Shimadzu UV-1700 spectrometer. The fluorescence spectra were measured using a fluorometer (LS-55, Perkin Elmer, USA). Average particle size and size distribution of the dots were determined by laser light scattering (LLS) with particle size analyzer (90 Plus, Brookhaven Instruments Co. USA) at a fixed angle of $90^{\circ}$ at room temperature. The morphology of dots was also studied by highresolution transmission electron microscope (HR-TEM, JEM-2010F, JEOL, Japan).

\subsection{Cell culture}

Cells were cultured in folate-free Dulbecco's Modified Eagle Medium (DMEM) containing 10\% fetal bovine serum and $1 \%$ penicillin streptomycin at $37{ }^{\circ} \mathrm{C}$ in a humidified environment containing $5 \% \mathrm{CO}_{2}$. Before experiment, the cells were pre-cultured until confluence was reached.

\subsection{In vitro fluorescence imaging}

MCF-7 breast cancer cells were cultured in the confocal imaging chambers (LAB-TEK, Chambered Coverglass System) at $37{ }^{\circ} \mathrm{C}$. After $80 \%$ confluence, the medium was removed and the adherent cells were washed twice with $1 \times$ 
PBS buffer. The AIE dots and Folate-AIE dots in FBS-free DMEM medium at $2 \mu \mathrm{M}$ of DTPEPBI were then added to the chambers, respectively. After incubation for $2 \mathrm{~h}$, the cells were washed three times with $1 \times$ PBS buffer and then fixed by $75 \%$ ethanol for 20 minutes, which were further washed twice with $1 \times$ PBS buffer and stained by DAPI for $10 \mathrm{~min}$. The cell monolayer was then washed twice with $1 \times$ PBS buffer and imaged by confocal laser scanning microscope (CLSM, Zeiss LSM 410, Jena, Germany) with imaging software (Olympus Fluoview FV1000) under the same experimental condition. The fluorescence signal from dots was collected at $543 \mathrm{~nm}$ excitation with a $560 \mathrm{~nm}$ longpass barrier filter. For flow cytometry analysis, two groups of cells were trypsinized and fixed in 75\% ethanol after incubation with AIE-dots and Folate-AIE dots, respectively. The cells were then suspended in $1 \times$ PBS buffer and the fluorescence intensities were analyzed using Cyan-LX (DakoCytomation). The histogram of each sample was obtained by counting 10,000 events ( $\lambda_{\mathrm{ex}}=488 \mathrm{~nm}, 750 \mathrm{~nm}$ longpass filter).

\subsection{Cytotoxicity studies}

The cytotoxicity of AIE dots and Folate-AIE dots was evaluated using MCF-7 cells through MTT assay. In brief, MCF-7 cells were seeded in 96-well plates (Costar, IL, USA) at a density of $2 \times 10^{4}$ cells $/ \mathrm{mL}$. After $48 \mathrm{~h}$ incubation, the medium was discarded and AIE dots and Folate-AIE dots in DMEM with various concentrations were added into each sample wells for further incubation at $37^{\circ} \mathrm{C}$, respectively. To eliminate the UV absorption interference of DTPEPBI at $570 \mathrm{~nm}$, the cells incubated with a series of AIE dots at the same doses but not post-treated by MTT were used as the control. After the designated time intervals, the sample wells were washed twice with $1 \times$ PBS buffer and $100 \mu \mathrm{L}$ of freshly prepared MTT $(0.5 \mathrm{mg} / \mathrm{mL})$ solution in culture medium was added into each well. After $3 \mathrm{~h}$ incubation in the incubator, the MTT medium solution was carefully removed, followed by addition of $100 \mu \mathrm{L}$ of DMSO into each well and the plate was gently shaken for $10 \mathrm{~min}$ at room temperature to dissolve all the precipitates formed. The absorbance of MTT at $570 \mathrm{~nm}$ was monitored by the microplate reader (Genios Tecan). Cell viability was expressed by the ratio of the absorbance of the cells incubated with fluorescent dot suspension to that of the cells incubated with culture medium only.

\subsection{In vivo fluorescence imaging}

All animal studies were performed in compliance with guidelines set by the Animal Care Committee at DrumTower Hospital. $0.1 \mathrm{~mL}$ of $\mathrm{H}_{22}$ cell suspension containing $5 \times 10^{6}-6 \times 10^{6}$ cells were injected subcutaneously to ICR mice (average body weight of $25 \mathrm{~g}$ ) at the left axilla. When the tumor volume reached a mean size of about $300 \mathrm{~mm}^{3}$, the mice were intravenously injected with $250 \mu \mathrm{L}$ of AIE dots and Folate-AIE dots, respectively, at the dye concentration of $4 \mathrm{mg} / \mathrm{kg}$ animal. Subsequently, the mice were anesthetized and placed on an animal plate heated to $37^{\circ} \mathrm{C}$. The biodistribution in mice was imaged using the Maestro in vivo fluorescence imaging system (CRi, Inc.). The light with a central wavelength at $523 \mathrm{~nm}$ was selected as the excitation source. In vivo spectral imaging from 560 to 900 $\mathrm{nm}(10 \mathrm{~nm}$ step) was conducted with an exposure time of $150 \mathrm{~ms}$ for each image frame. Auto-fluorescence was removed by using the spectral unmixing software. Scans were carried out at 2, 8 and $24 \mathrm{~h}$ post-injection.

\section{Results and discussion}

The fluorescent dots with aggregation-induced emission feature were synthesized through a nanoprecipitation method [12]. Using a mixture of 1,2-distearoyl-sn-glycero-3phosphoethanolamine- $N$-[methoxy(polyethylene glycol)2000] (DSPE-PEG ${ }_{2000}$ ) and DSPE-PEG ${ }_{5000}$-Folate as the encapsulation matrix, the fluorescent dots with good biocompatibility and surface folic acid groups for folatereceptor overexpressed cancer cell targeting were obtained. Folate-AIE dots and AIE dots represent fluorescent dots that were formulated with encapsulation matrix containing $50 \%$ and $0 \%$ of DSPE-PEG ${ }_{5000}$-Folate, respectively. During formation, the hydrophobic DSPE segments tend to be embedded into the hydrophobic core with DTPEPBI while the hydrophilic PEG-folate chains extend into the aqueous phase.

Laser light scattering (LLS) result suggests that the volume average hydrodynamic diameter of Folate-AIE dots is $52 \pm 3 \mathrm{~nm}$ (Figure 1(a)), which is similar to that of the AIE dots $(49 \pm 2 \mathrm{~nm})$. The morphology of Folate-AIE dots were studied by high-resolution transmission electron microscopy (HR-TEM), which is shown as the inset in Figures 1(a). The black dots suggest that the spherical Folate-AIE dots can be clearly distinguished due to the high electron density of DTPEPBI molecules. Figure 1(b) shows the UV-Vis absorption and photoluminescence (PL) spectra of Folate-AIE dots in water, suggesting that the Folate-AIE dots have an emission maximum at $734 \mathrm{~nm}$, which is similar to that of AIE dots in water $(732 \mathrm{~nm})$. The quantum yield of Folate-AIE dots in water is measured to be $3 \%$, using rhodamine $6 \mathrm{G}$ in ethanol as the standard. The intense emission of Folate-AIE dots in FR/NIR window will greatly benefit in vivo experiments with deep tissue penetration depth and minimized autofluorescence interference.

MCF-7 breast cancer cells that are known to have high folate receptor expression level in cell membrane were chosen to evaluate the targeting ability of Folate-AIE dots over AIE dots without surface folic acid groups [13]. The effect of surface folic acid groups on internalization efficiency of the dots by MCF-7 breast cancer cells was investigated using confocal laser scanning microscopy. Figures 


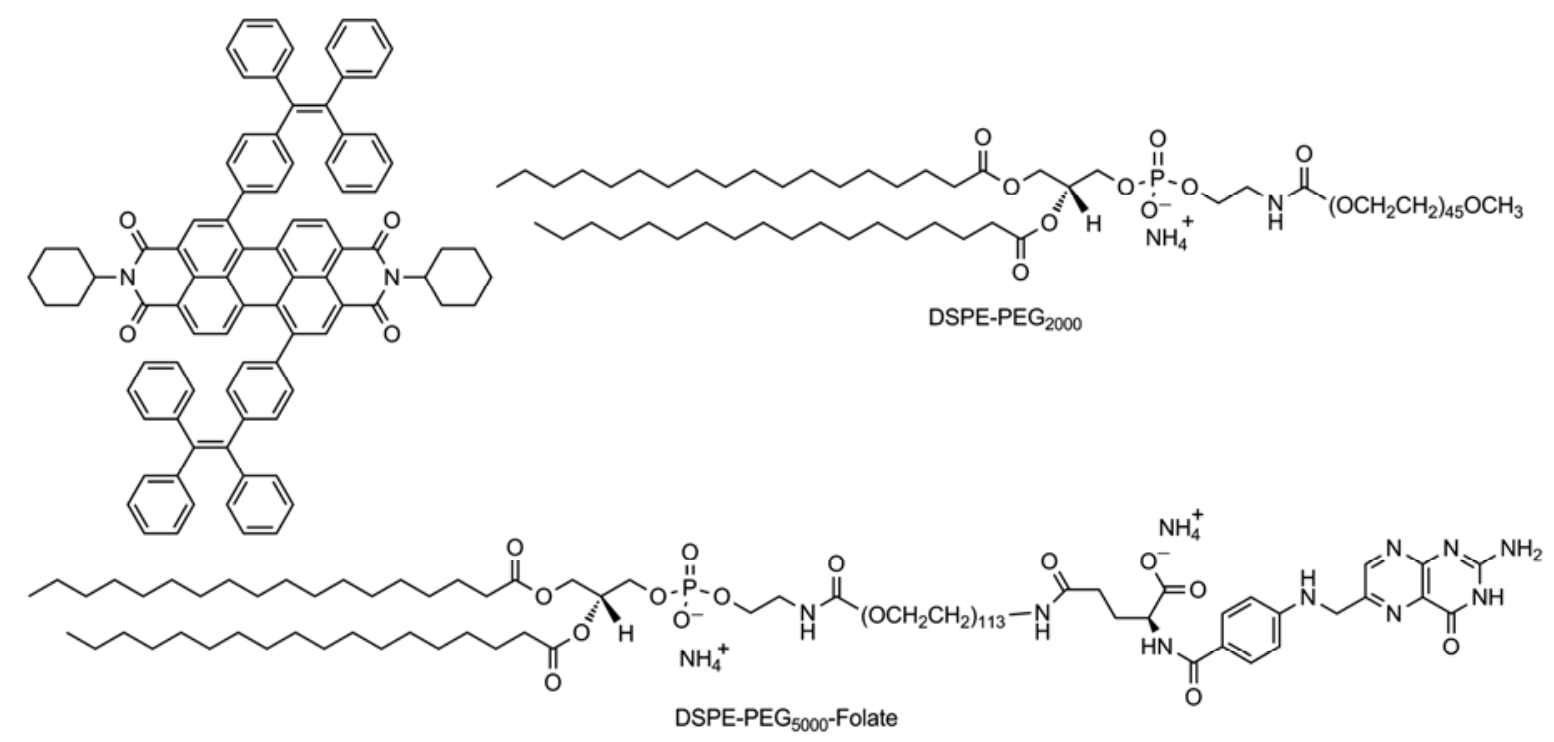

Scheme 1 Chemical structures of DTPEPBI, DSPE-PEG 2000 and DSPE-PEG ${ }_{5000}$-Folate.
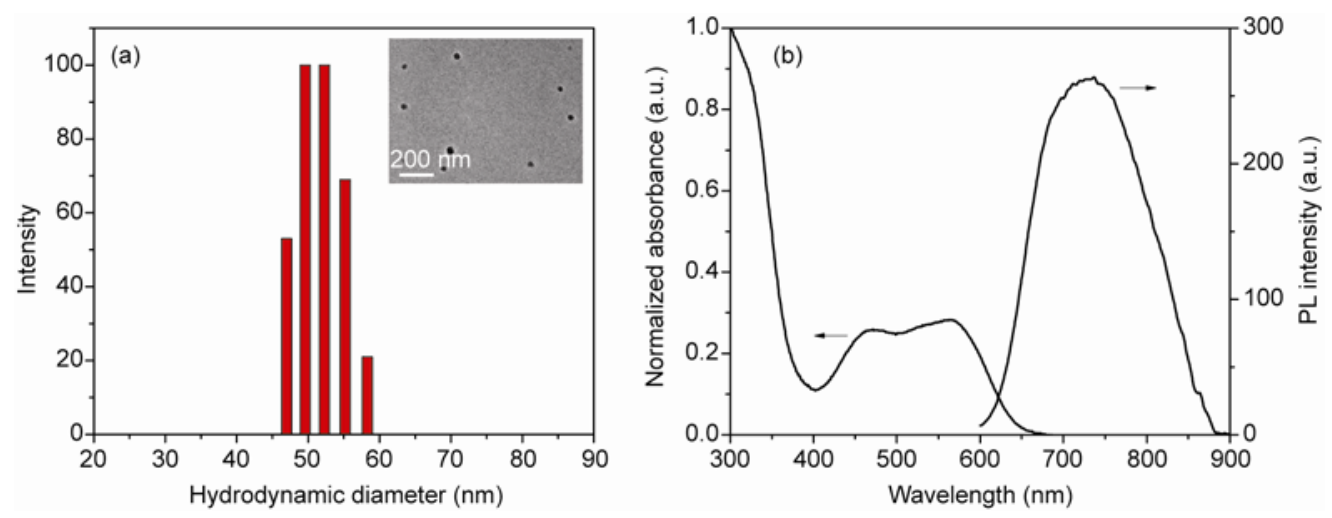

Figure 1 (a) Particle size distribution of Folate-AIE dots in water studied by laser light scattering. The inset shows HR-TEM image of Folate-AIE dots; (b) UV-Vis absorption and PL spectra of Folate-AIE dots in water (excited at $543 \mathrm{~nm}$ ).

2(a) and (b) show the confocal images of MCF-7 breast cancer cells after incubation with AIE dots and Folate-AIE dots in freshly prepared culture medium for $2 \mathrm{~h}$ at DTPEPBI concentration of $2 \mu \mathrm{M}$, respectively. The cell nuclei were stained with 4',6-diamidino-2-phenylindole (DAPI). These images were taken upon excitation at $543 \mathrm{~nm}$ with a $560 \mathrm{~nm}$ longpass barrier filter. It should be noted that no auto fluorescence from the cell itself can be detected under the same experimental condition (Figure 2(c)). Comparison between Figures 2(a) and (b) suggests that the fluorescence intensity from cell cytoplasm after incubation with Folate-AIE dots is higher than that after incubation with AIE dots. The higher fluorescence intensity of MCF-7 cancer cells in Figure 2(a) as compared to that in Figure 3(b) suggests that more fluorescent dots are internalized into the cells due to specific interactions between folic acid on Folate-AIE dot surface and folate receptors in the cancer cell membrane, which should favor folate receptor-mediated endocytosis $[14,15]$. The flow cytometry histograms of MCF-7 cancer cells after incubation with AIE dots and Folate-AIE dots are shown in Figure 2(d), indicating that the average fluorescence intensity of each cell incubated with Folate-AIE dots is $\sim 1.3$ times higher as compared to that upon incubation with AIE dots. In addition, the cytotoxicity of both AIE dots and Folate-AIE dots was evaluated through methylthiazolyldiphenyltetrazolium bromide (MTT) assays. The results suggest that the metabolic viability of MCF-7 cancer cells after incubation with fluorescent dots at 2, 4 and $8 \mu \mathrm{M}$ of DTPEPBI for $48 \mathrm{~h}$ remains above $90 \%$, suggesting both AIE dots and Folate-AIE dots are of low cytotoxicity (Figure 2(e)).

The performance of Folate-AIE dots in in vivo imaging was further investigated on a folate receptor-overexpressed tumor-bearing mouse model, using AIE dots without folic acids as the control. The animal model was established through subcutaneous inoculation of murine hepatic $\mathrm{H}_{22}$ cancer cells into the right flank of each mouse. When the tumor volume reached about $300 \mathrm{~mm}^{3}$, the mice were in- 

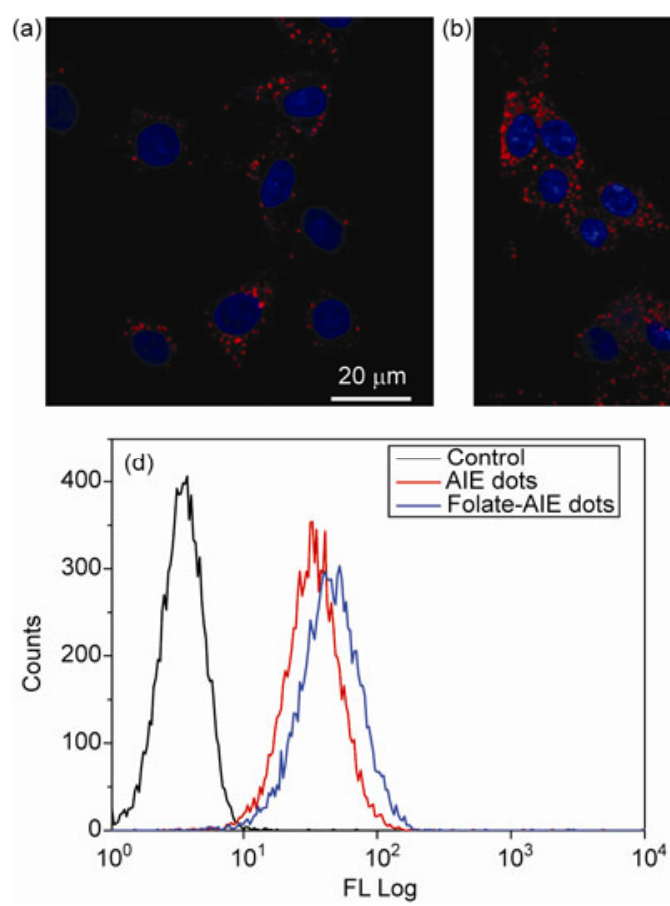
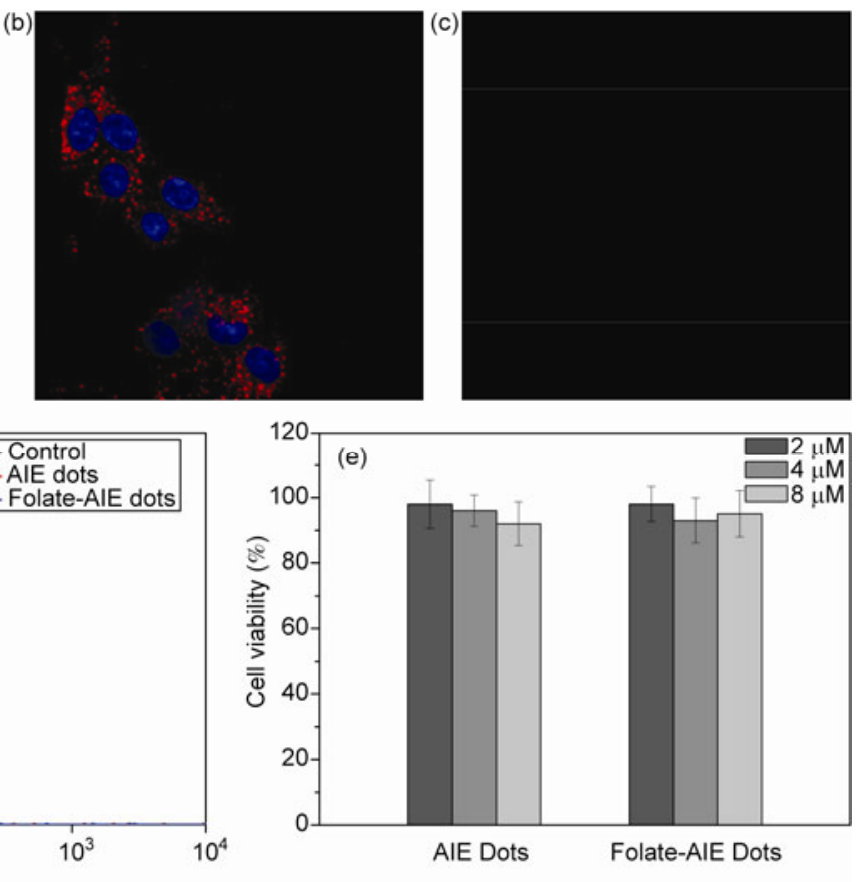

Figure 2 Confocal images of MCF-7 cancer cells after incubation with AIE dots (a) and Folate-AIE dots (b) for $2 \mathrm{~h}$ at $37{ }^{\circ} \mathrm{C}([\mathrm{DTPEPBI}]=2 \mu \mathrm{M})$. The fluorescence signal was recorded under excitation at $543 \mathrm{~nm}$ with a $560 \mathrm{~nm}$ longpass barrier filter. The blue signal indicates cell nuclei stained by DAPI; (c) confocal image of the pristine cells without incubation fluorescent dots. All the images share the same scale bar; (d) flow cytometry histograms of pure MCF-7 cells (control) and cells treated with AIE dots and Folate-AIE dots; (e) metabolic viability of MCF-7 cells after 48 h incubation with AIE dots and Folate-AIE dots at the DTPEPBI concentrations of 2,4 and $8 \mu \mathrm{M}$, respectively.

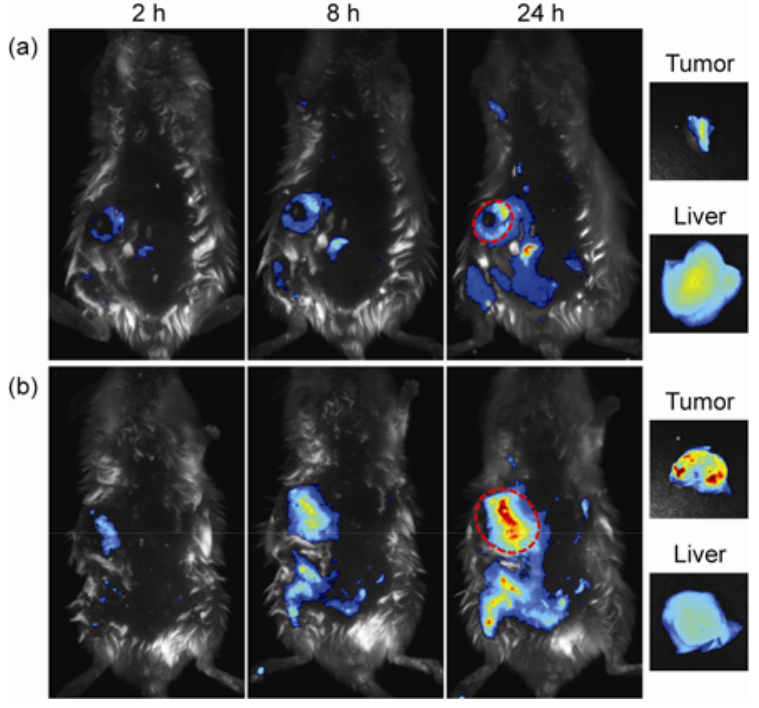

Figure 3 In vivo fluorescence imaging of $\mathrm{H}_{22}$ tumor-bearing mice after intravenous injection of AIE dots (a) and Folate-AIE dots (b), respectively. The red circle indicates the tumor site.

travenously injected with AIE dots and Folate-AIE dots through tail vain, respectively. After designated time intervals upon injection, the mice were imaged by a Maestro EX in vivo fluorescence imaging system with a central wavelength at $523 \mathrm{~nm}$ as the excitation source. Figure 3(a) shows the in vivo distribution of AIE dots without folic acids in the tumor-bearing mouse at 2,8 and $24 \mathrm{~h}$ post-injection. The FL intensities are indicated with different colors, and the order of red, orange, yellow, green, and blue refers to a successive decrease in intensity. Obvious fluorescence signal is observed in the area of tumor tissue at all the time points, suggesting that AIE dots have efficiently accumulated in tumor through enhanced permeability and retention (EPR) effect [16]. In addition, strong fluorescence from the liver region is also observed, which implicates that a portion of fluorescent dots in the blood circulation tend to be enriched in the liver, due to reticuloendothelial system (RES) uptake [17]. On the other hand, the specific tumor targeting ability of Folate-AIE dots is also evaluated on the same tumor-bearing mouse model (Figure 3(b)). Much higher fluorescence intensity is shown in the tumor tissue of Folate-AIE dot-treated mouse as compared to that of AIE dot-treated mouse at all the time points, demonstrating the specific targeting ability of Folate-AIE dots to the tumor that contains folate receptor-overexpressed cancer cells in a living body. Noteworthy is that the ratio between fluorescence intensity from the tumor tissue and that from the liver tissue upon administration of Folate-AIE dots is significantly enhanced as compared to that upon administration of AIE dots. These results clearly indicate that Folate-AIE dots can be used as an effective fluorescent probe for in vivo tumor diagnosis with high specificity and good fluorescence contrast. 


\section{Conclusion}

In this work, we successfully synthesized FR/NIR organic fluorescent dots using luminogens with aggregation-induced emission (AIE) feature as the core and biocompatible DSPE-PEG derivatives as the encapsulation matrix. Through mixing DSPE-PEG-Folate with DSPE-PEG, Folate-AIE dots with surface folic acid groups were obtained to facilitate targeted imaging studies. In vitro studies using MCF-7 cancer cells with high folate receptor overexpression as a model suggest that the folic acid-functionalized organic dots have higher living cell internalization efficiency as compared to AIE dots without surface folic acids, due to folate-receptor mediated endocytosis. The targeting ability of Folate-AIE dots to folate receptor-overexpressed tumor was also investigated on an animal model, indicating that Folate-AIE dots preferentially accumulated at the tumor site and the emission profile of the dots in FR/NIR region is greatly beneficial to in vivo fluorescence imaging. These results demonstrate the great potential of DTPEPBI-based Folate-AIE dots in cancer diagnosis with high specificity and good fluorescence contrast for in vivo studies. In future, such AIE dots with incorporation of therapeutic reagents will provide a promising platform to construct multifunctional dots for tumor diagnosis and treatment.

We are grateful to the support from the Agency for Science Technology and Research Joint Council and Institute of Materials Research and Engineering of Singapore (IMRE/13-8P1104), the Singapore National Research Foundation (R-279-000-390-281), the Research Grants Council of Hong Kong (603509, HKUST2/CRF/10, 604711, N_HKUST620/11), and the Guangdong Innovative Research Team Program (201101C0105067115).

1 Hong G, Lee JC, Robinson JT, Raaz U, Xie L, Huang NF, Cooke JP, Dai $H$. Multifunctional in vivo vascular imaging using near-infrared II fluorescence. Nat Med, 2012, 18: 1841-1846

2 Smith AM, Duan H, Mohs AM, Nie, S. Bioconjugated quantum dots for in vivo molecular and cellular imaging. Adv Drug Deliv Rev, 2008, 60: $1226-1240$
3 Lewinski N, Colvin V, Drezek R. Cytotoxicity of nanoparticles. Small, 2008, 4: 26-49

4 Liu YS, Sun YH, Vernier PT, Liang CH, Chong SYC, Gundersen MA. pH-sensitive photoluminescence of $\mathrm{CdSe} / \mathrm{ZnSe} / \mathrm{ZnS}$ quantum dots in human ovarian cancer cells. J Phys Chem C, 2007, 111: 2872-2878

5 Qin AJ, Zhang Y, Han N, Mei J, Sun J, Fan W Tang BZ. Preparation and self-assembly of amphiphilic polymer with aggregation-induced emission characteristics. Sci China Chem, 2012, 55: 772-778

6 Hong Y, Lam JWY, Tang BZ. Aggregation-induced emission: Phenomenon, mechanism and applications. Chem Commun, 2009, 4332-4353

7 Yu Y, Hong Y, Feng C, Liu J, Lam JWY, Faisal M, Ng KM, Luo KQ, Tang BZ. Synthesis of an AIE-active fluorogen and its application in cell imaging. Sci China Ser B-Chem, 2009, 52: 12-19

8 Li HK, Mei J, Wang J, Zhang S, Zhao Q, Wei Q, Qin A, Sun J, Tang BZ. Facile synthesis of poly(aroxycarbonyltriazole)s with aggregationinduced emission characteristics by metal-free click polymerization. Sci China Chem, 2011, 54: 611-616

9 Hong Y, Lam JWY, Tang BZ. Aggregation-induced emission. Chem Soc Rev, 2011, 40: 5361-5388

10 Li K, Qin W, Ding D, Tomczak N, Geng J, Liu R, Liu J, Zhang X, Liu H, Liu B, Tang BZ. Photostable fluorescent organic dots with aggregation-induced emission (AIE dots) for noninvasive long-term cell tracing. Sci Rep, 2013, 3: 1150

11 Zhao Q, Zhang S, Lu Y, Mei J, Chen S, Lu P, Qin A, Ma Y, Sun JZ, Tang BZ. Tetraphenylethenyl-modified perylene bisimide: Aggregation-induced red emission, electrochemical properties and ordered microstructures. J Mater Chem, 2012, 22: 7387-7394

12 Prashant C, Dipak M, Yang CT, Chuang, KH, Jun D, Feng SS. Superparamagnetic iron oxide-loaded poly(lactic acid)-D-alphatocopherol polyethylene glycol 1000 succinate copolymer nanoparticles as MRI contrast agent. Biomaterials, 2010, 31: 5588-5597

13 Sudimack J, Lee RJ. Targeted drug delivery via the folate receptor. Adv Drug Deliv Rev, 2000, 41: 147-162

14 Low PS, Henne WA, Doorneweerd DD. Light-controlled release of caged doxorubicin from folate receptor-targeting PAMAM dendrimer nanoconjugate. Acc Chem Res, 2008, 41: 120-129

15 Geng J, Li K, Ding D, Zhang X, Qin W, Liu J, Tang BZ, Liu B. Lipid-PEG-Folate encapsulated nanoparticles with aggregation induced emission characteristics: Cellular uptake mechanism and two-photon fluorescence imaging. Small, 2012, 8: 3655-3663

16 Liu Z, Chen K, Davis C, Sherlock S, Cao Q, Chen XY, Dai HJ. Drug delivery with carbon nanotubes for in vivo cancer treatment. Cancer Res, 2008, 68: 6652-6660

17 Li SD, Huang L. Factors affecting the clearance and biodistribution of polymeric nanoparticles. Mol Pharmaceutics, 2008, 5: 496-504 